\title{
Enlightening macromolecular structure-function relationship with proteopedia
}

\author{
Jaime Prilusky ${ }^{1}$, Joel Sussman ${ }^{2}$ \\ ${ }^{1}$ Life Sciences Core Facilities, Weizmann Institute Of Science, Rehovot, Israel, ${ }^{2}$ Department of Structural Biology, Weizmann \\ Institute of Science, Rehovot 761000, Israel \\ E-mail: jaime.prilusky@weizmann.ac.il
}

Students and scientists are now able to access 3D images of biomacromolecules both in journal and on the web. However, rather than just relying on text and $2 \mathrm{D}$ images to try to understand the function of biomacromolecular structures, a collaborative website called Proteopedia1,2 is a free resource, which links written information \& 3D structural images. This wiki web site, http://proteopedia.org displays protein structures \& other biomacromolecules interactively. These images are surrounded by descriptive text containing hyperlinks that change the appearance (e.g., views, representations, colors or labels) of the adjacent 3D structure to reflect the concepts discussed in the text. This makes the complex structural information readily accessible and comprehensible, even to non-structural biologists. Using Proteopedia, one can easily create descriptions of biomacromolecules linked to their 3D structure, e.g., see a page on the ribosome structure/function, http://proteopedia.org/w/Ribosome. Pages can be viewed on PCs, MACs \& LINUX computers and even on iPads (that do not have JAVA), via the molecular viewer JSmol3, e.g., a page on HIV-1 protease, http://proteopedia.org/w/HIV-1_protease.

Content is being added by Proteopedia's >3,500 users, in 60 different countries, in a dozen different languages, including Russian, Chinese, Arabic, \& Hebrew: http://proteopedia.org/w/1eve_(Arabic). A number of journals \& book publishers are using Proteopedia to complement their printed and web papers using Proteopedia's "Interactive 3D Complements" (I3DCs) see, e.g., http://proteopedia.org/w/Journal:Molecular_Cell:1.

Pages for each of the $>127,000$ entries in the PDB have been automatically created with 'seed' information, and are both intrinsically useful and 'primed' for expansion by users. Scientists \& students are invited to request a Proteopedia user account, at no cost, in order to edit existing pages \& to create new ones, see: http://proteopedia.org/w/Special:RequestAccount.

1. Hodis, E., Prilusky, J., Martz, E., Silman, I., Moult, J. \& Sussman, J. L. (2008). Genome Biol 9, R121.

2. Prilusky, J., Hodis, E., Canner, D., Decatur, W. A., Oberholser, K., Martz, E., Berchanski, A., Harel, M. \& Sussman, J. L. (2011). J Struct Biol 175, 244.

3. Hanson, R. M., Prilusky, J., Renjian, Z., Nakane, T. \& Sussman, J. L. (2013). Israel J Chem 53, 207.

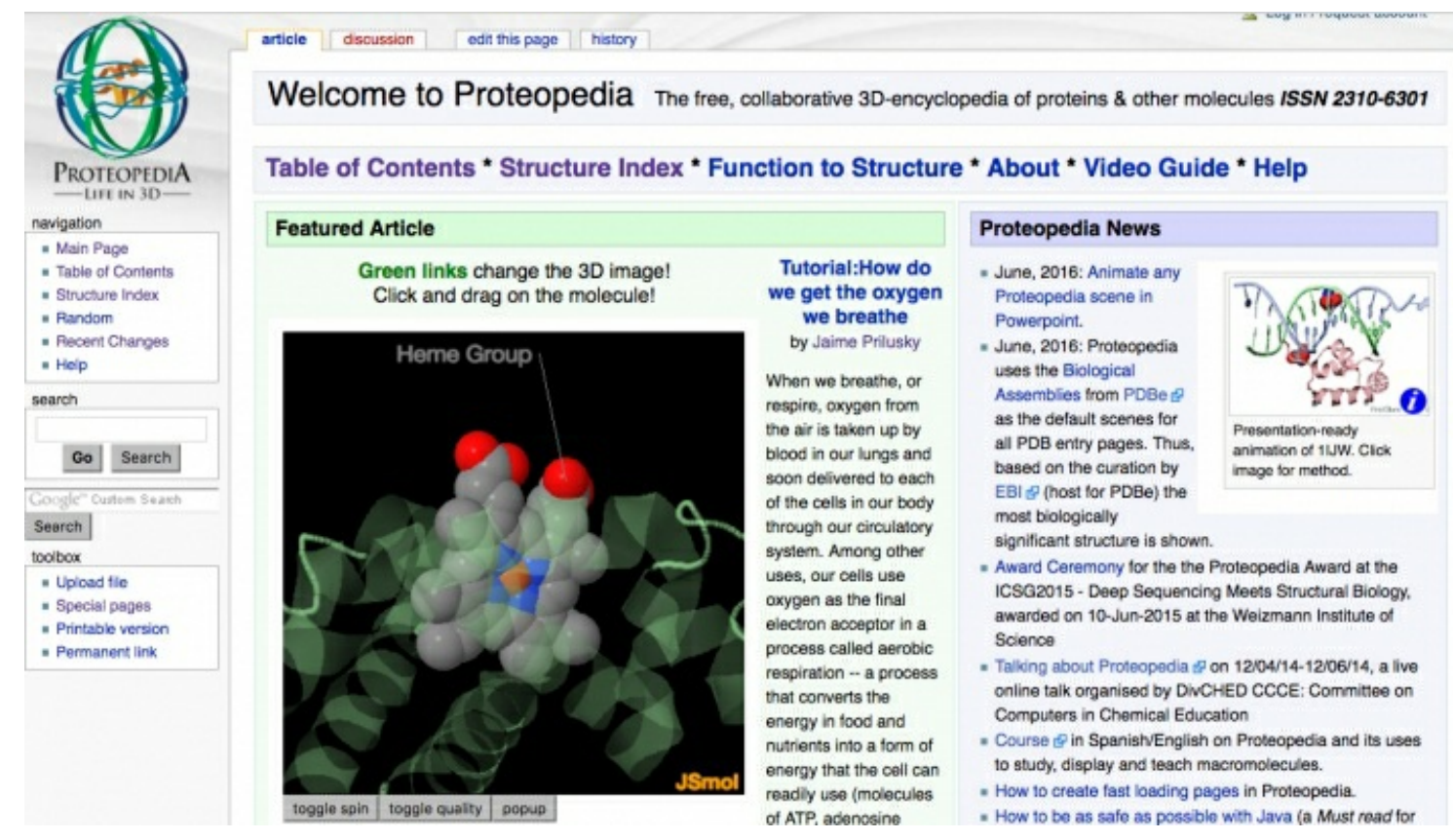

Keywords: molecular visualisation, teaching protein structure function, interactive educational media 